\title{
Forced-choice technique: Inter-alternative comparisons
}

WILLIAM H. WATKINS AND HERBERT J. CLARK, AEROSPACE MEDICAL RESEARCH LABORATORIES, AEROSPACE MEDICAL DIVISION, AIR FORCE SYSTEMS COMMAND, Wright-Patterson Air Force Base, Ohio

In certain listening tests, where Ss were required to identify which sequentially presented alternative matched a standard stimulus, $S$ 's accuracy varied monotonically with the sequential position of the correct alternative. If a trial consisted of the standard stimulus followed by four alternatives, $S$ was most likely to identify the stimulus correctly if it was the first alternative, etc. (Conversely, if the standard was presented after the four alternatives.) Our research suggests, however, that if the standard is presented before a long series of 4-alternative trials rather than before each trial, $S$ 's accuracy does not vary with the position of the correct alternative.

This study was concerned with response behavior in 4 alternative forced-choice (4AFC) recognition and detection tests. Data examined represented two different applications of 4AFC technique: (1) where a sample stimulus preceded each trial of four alternatives, and (2) where a sample occurred only at the beginning of a series of $48 \mathrm{FC}$ trials. Investigation centered on the distributions of correct responses and frequency of selection among the 4-alternatives.

The FC method of data collection is in wide use and has been highly recommended. The intent of the analysis was to provide additional information for judging the suitability of the method.

Talker Identification Experiments. Clarke, Becker, \& Nixon (1966) reported that correct identification of voices in a 4AFC test declined monotonically in alternative 1-2-3-4-order when a sample of the target voice preceded the presentation of four alternatives. When the target voice followed the presentation of alternatives, correct identification declined monotonically in reverse order.

Watkins, Schjelderup, \& Busch (unpublished) conducted a 4AFC talker identification test in which a sample utterance preceded four alternative utterances to comprise each "match-thesample" trial. Figure 1 shows the salient findings of the analysisaccuracy and response frequency declined from Alternative 1 through Alternative 4. The $L$ test of Page was chosen to determine the statistical significance of these apparently monotonic declines. $L$ values obtained were statistically significant in both cases $(\mathrm{p}<.01)$.

The upper curve of Figure 1 agrees with comparable data reported by Clarke et al. Response frequency ("popularity") of the alternatives may be seen in the lower curve of Figure 1 to have varied directly with accuracy. The target voices were actually distributed on a quasi-random, equal basis within the 36-item test.

Signal Detection Experiments. Interest developed in the question of whether or not the response frequency and correctness pattern obtained above would also be found in other 4AFC data. Data from three previously completed experiments (Watkins \& Feehrer, 1965; Watkins, 1966; Watkins \& Schjelderup, 1967) were examined. Relatively simple visual or auditory stimuli had been employed. In contrast to the talker identification task in which the sample stimulus was presented before each FC trial, here, a sample stimulus was presented only at the beginning of a series of FC trials. The sample (tone segment or light flash) was displayed against a quiet background prior to the first trial of a run of 48

\section{TALKER IDENTIFICATION}
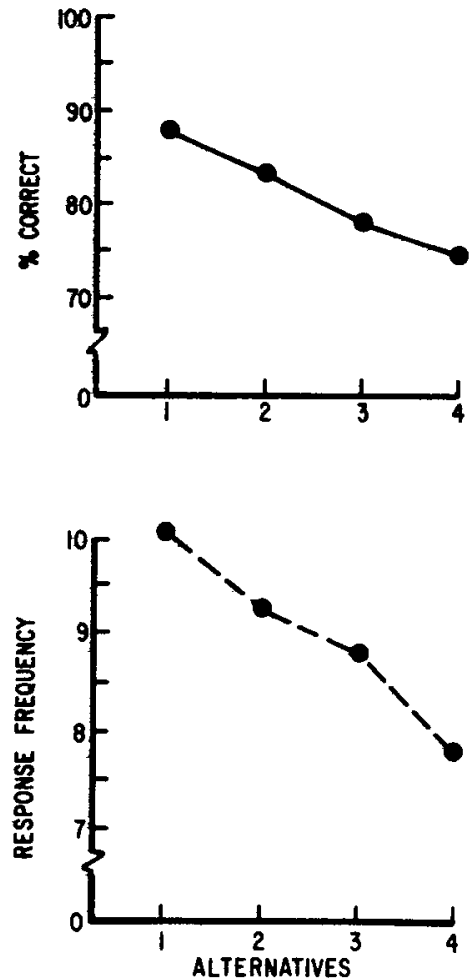

Fig. 1. Means (6 Ss) for percentage correct (Upper Graph) and response frequency (Lower Graph), according to FC interval $1 \ldots 4$, on a Talker Identification Test (Watkins et al, unpublished).
SIGNAL DETECTION
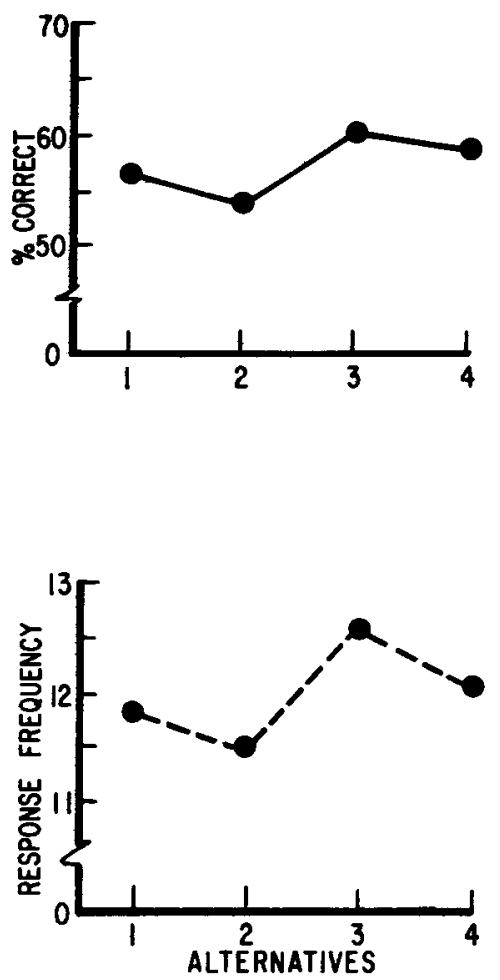

Fig. 2. Means (8 Ss) for percentage correct (Upper Graph) and response frequency (Lower Graph), according to FC interval $1 \ldots 4$ on a Signal Detection Test (Watkins, 1966). 
trials. Within a run, the signal to be detected was always the same and was presented at one of four temporal alternatives against a noisy background.

Figure 2 typifies the results of analyses for each of the three experiments in showing only negligible (and non-significant) differences among alternatives in both percentage correct and frequency of selection. Interactions of the "Alternative" variable with experimental conditions were examined and found to be non-significant.

\section{DISCUSSION}

When a sample stimulus preceded each FC trial (talker identification), there was a significant decline in recognition accuracy and response frequency from Alternative 1 through Alternative 4. Clarke et al (1966, p.ll) imply that the decline in accuracy may be due to the increasing effects of interference as the number of alternatives between sample and correct alternative increases. Since Clarke found monotonic declines in 1-2-3-4 or 4-3-2-1 order, depending upon whether the sample preceded or followed the alternatives, his explanation appears reasonable. However, when a sample stimulus was presented only preceding a series of FC trials (signal detection), neither detection nor response frequency varied significantly among alternatives. In short, the declines found in the talker identification tasks are not characteristic of all $4 \mathrm{AFC}$ tasks.
The number and kinds of differences in procedure for the two FC applications studied preclude any defensible inference as to why interalternative differences were found in one instance and not in the other. An implication of these results is that $\mathbf{E}$ should insure adequately balanced representation of each experimental condition across all alternatives in $4 \mathrm{AFC}$ identification tasks when a sample stimulus is to precede each trial.

\section{REFERENCES}

CLARKE, F. R., BECKER, R. W., \& NIXON, J. C. Characteristics that determine speaker recognition. USAF ESD TR 66-636, 1966.

WATKINS, W. H. Photic facilitation of tonal signal detection in a forced-choice situation. Psychon. Sci, 1966, 6, 477-478.

WATKINS, W. H., \& FEEHRER, C. E. Acoustic facilitation of visual detection. J. exp. Psychol., 1965, 70, 332-333.

WATKINS, W. H., \& SCHJELDERUP, J. R. Effects of temporal variation of auxiliary light stimuli upon detectability of tonal signals. Percept. \& Psychophys, 1967, 2, 317-322.

\section{NOTE}

1. This paper has been identified by Aerospace Medical Research Laboratories as AMRL-TR-68-18. Further reproduction is authorized to satisfy the needs of the U.S. Government. 